Marius Roth

\title{
Zu den Titeln, Kurztiteln und Abkürzungen von Erlassen
}

Die Praxis bei der Verwendung von Titeln, Kurztiteln und Abkürzungen ist sehr unterschiedlich. Nachfolgend seien ein paar Gedanken aus der Sicht eines Anwenders beschrieben, der sich täglich mit diesem Thema beschäftigt.

Beitragsart: Werkstattberichte

Zitiervorschlag: Marius Roth, Zu den Titeln, Kurztiteln und Abkürzungen von Erlassen, in: LeGes 31 (2020) 3 


\section{Inhaltsübersicht}

1. Einführung

2. Zum eigentlichen Erlasstitel

3. Praxis der Titelgebung für Langtitel

4. Abkürzungen und Kurztitel

4.1. Allgemeine Betrachtung

4.2. Abkürzungen

4.3. Kurztitel

5. Titel von Geschäften/Publikationen in der chronologischen Gesetzessammlung

6. Die Änderung von Titeln und Abkürzungen

6.1. Allgemeine Betrachtung

6.2. Änderungen von Titeln

6.3. Änderung von Abkürzungen

7. Zitierung von Erlassen

8. Empfehlungen

\section{Einführung}

[1] Jeder Erlass verfügt mindestens über einen Titel, manche auch über einen Kurztitel und/oder eine Abkürzung. In der Praxis versucht man diese Titelelemente immer «gleich» zu gestalten; ein Blick in die systematischen Sammlungen von Bund und Kantonen zeigt aber, dass dies eher schlecht als recht gelingt.

\section{Zum eigentlichen Erlasstitel}

[2] Der Erlasstitel ist der «Langtitel», d. h. derjenige Titel, der nicht in Klammern gesetzt wird und am Anfang des Erlasses steht.

[3] Für den Bund ist der Titel in den Gesetzestechnischen Richtlinien (GTR) ${ }^{1}$ wie folgt definiert (vgl. Rz. 3):

Der Erlasstitel muss den Erlassgegenstand so spezifisch benennen, dass Verwechslungen mit anderen Erlassen ausgeschlossen sind, und gleichzeitig möglichst kurz sein. Aus dem Erlasstitel müssen Erlassform und Regelungsgegenstand sowie bei bestimmten Erlassformen das erlassende Organ hervorgehen. Damit der Erlasstitel zitierbar bleibt, muss vermieden werden, den Regelungsgegenstand des Erlasses in allen Details wiederzugeben.

[4] Die Kantone definieren den Titel ähnlich, wie die folgenden Beispiele zeigen:

[5] Kanton Aargau²:

Der Erlasstitel gibt den charakteristischen Inhalt des Erlasses wieder und ist in der Regel mit einem Kurztitel und einer Abkürzung zu versehen.

1 Gesetzestechnische Richtlinien (www.bk.admin.ch $>$ Dokumentation $>$ Rechtsetzungsbegleitung).

2 Vgl. Formalien der Rechtsetzung vom 6. Dez. 2011, Ziff. 2.2. 
Der Titel setzt sich zusammen aus der Bezeichnung der Erlassart (Gesetz, Dekret, Verordnung), einer möglichst kurzen Umschreibung des im Erlass geregelten Gegenstands, dem Kurztitel und gegebenenfalls der Abkürzung. Ein Kurztitel ist nur dann zu wählen, wenn er auch Sinn macht. Insbesondere bei sehr kurzen Erlasstiteln kann der Kurztitel weggelassen werden.

[6] Kanton Zürich ${ }^{3}$ :

Der Titel des Erlasses umschreibt seinen zentralen Gegenstand (sein Hauptthema) kurz und prägnant. Unter Umständen muss dabei eine Auswahl unter den Gegenständen des Erlasses getroffen werden. Im Titel muss ferner die Erlassform erkennbar sein. Erlasse, die der Einführung von Bundesrecht dienen, erhalten die Bezeichnung Einführungsgesetz bzw. Einführungsverordnung.

Kann der Erlassgegenstand in einem einzigen Begriff umschrieben werden, soll der Erlasstitel als zusammengesetztes Wort ausgestaltet werden.

[7] Im Titel müssen somit primär die Erlassform sowie der Hauptregelungsgegenstand bzw. das Hauptthema zum Ausdruck kommen. Nebenthemen und partikuläre Aspekte einer Rechtsnorm sollen zugunsten der Prägnanz nicht im Titel in Erscheinung treten. Auch sollte der Titel nicht versuchen, den jeweiligen Erlass zusammenzufassen, wie dies beispielsweise der Fall ist bei der «Verordnung über die Möglichkeit gewisser Geschäfte, an zwei Sonn- und Feiertagen pro Jahr bewilligungsfrei Arbeitnehmende zu beschäftigen» (FR 940.15²), dem "Arrêté ordonnant la sonnerie des cloches pour fêter le jour anniversaire de la fondation de la Confédération suisse le $1^{\text {er }}$ août» (VD 111.15.1) oder dem «Regierungsratsbeschluss betreffend Liste der als potentiell gefährlich eingestuften Hunderassen und deren Kreuzungen gemäss $\S 14$ Abs. 2 des Gesetzes betreffend das Halten von Hunden (Hundegesetz)» (BS 365.101).

[8] Ein weiteres Ziel des Titels besteht in der Vermeidung von Verwechslungen mit anderen Erlassen. Mit anderen Worten dient der Erlasstitel dazu, einen Erlass (möglichst) eindeutig benennen und zitieren zu können. Aus diesem Grund sind namentlich Titeländerungen problematisch (vgl. dazu unten Ziff. 6). In den systematischen Sammlungen finden sich aber auch Titel, die kaum Aufschluss darüber geben, worum es im Erlass selber gehen könnte (vgl. z. B. SR 0.632.401.7: Schlussakte oder ZH 831.3: Zusatzleistungsgesetz).

[9] Nach Ansicht des Autors gehört der Titel nicht zum normativen Inhalt eines Erlasses. Dies bedeutet, dass daraus keine Rechte oder Pflichten abgeleitet werden können, und dass ein ungenauer oder irreführender Titel nicht dazu führen kann, dass die darin festgesetzten Rechtsnormen keine Geltung beanspruchen können, soweit das jeweilige Rechtsetzungsverfahren eingehalten wurde.

[10] Zusammengefasst hat der Titel einen rein beschreibenden Charakter und dient in erster Linie dazu, den Rechtsuchenden sowie den Rechtsanwenderinnen und Rechtsanwendern das Auffin-

Vgl. Richtlinien der Rechtsetzung vom 21. Dez. 2005, Rz. 27 ff.

4 Kantonale Erlasse werden in diesem Beitrag immer mit dem Kantonskürzel und der systematischen Nummer zitiert, Bundeserlasse entweder mit «SR» oder mit «CH». 
den und Zitieren einer Rechtsnorm zu erleichtern. Deshalb spielt es eigentlich auch eine untergeordnete Rolle, was konkret der Inhalt eines Titels ist.

\section{Praxis der Titelgebung für Langtitel}

[11] Seit Beginn der modernen Erlasspublikation (ab der Zeit der Mediation) besteht die Praxis, dass zuerst die Rechtsform (Gesetz, Verordnung etc.) und anschliessend das Thema genannt wird. Auf Deutsch wurde früher als Verbindungswort zwischen Rechtsform und Thema meistens der Begriff «betreffend» verwendet. Heute wird indessen fast ausschliesslich das Wort «über» gebraucht. $^{5}$

[12] Gleichzeitig gibt es in den Kantonen mit Amtssprache Deutsch seit jeher die Gepflogenheit, bestimmte Erlasse nur mit einem «Kurztitel» (vgl. dazu auch unten Ziff. 4.3) zu versehen, ${ }^{6}$ wie z. B. «Schulgesetz» (vgl. z. B.: ZG 412.11, AG 401.100, SH 410.100), «Baugesetz» (so z. B. BE 721.0, OW: 710.1; AI: 700.000) oder «Steuergesetz» (vgl. ZH 631.1; AG 651.100 oder SZ 172.200).

[13] Teilweise verfügen Titel auch über Fussnoten. Dabei kann es sich einerseits um sog. Änderungsfussnoten handeln, die die Leserin oder den Leser auf Änderungen des Langtitels bzw. des Kurztitels oder der Abkürzung hinweisen. Dies ist vor allem bei Erlassen des Bundes oder jener Kantone der Fall, die noch keine Änderungstabellen verwenden. ${ }^{7}$ Andererseits beinhalten sog. «Informationsfussnoten» allgemeine Hinweise zum betreffenden Erlass, wie z. B. Fundstellen in der Chronologischen Sammlung, Genehmigungsvermerke oder Hinweise auf Materialien und Revisionen. ${ }^{8} \mathrm{Zu}$ dieser Kategorie zählt auch die «Stern-Fussnote», die der Bund oft in den französischen Sprachfassungen anbringt, jeweils mit dem identischen Inhalt: «Les termes désignant des personnes s'appliquent également aux femmes et aux hommes». ${ }^{9}$ Solche Fussnoten stellen immer eine Verlegenheitslösung des Erlassredaktors dar, die dazu dient, Informationen wiederzugeben, für die kein geeigneter Ort gefunden werden konnte. Grundsätzlich sollten solche Hilfskonstrukte vermieden werden: Fussnoten enthalten in der Regel weiterführende Erklärungen zu einem Wort oder einer Passage und gehören technisch zum Element, das sie näher beschreiben. Werden solche Fussnoten in Erlasstiteln verwendet, können sie jedoch nicht dem jeweiligen Titel zugerechnet werden und werden auch nicht zusammen mit diesem zitiert.

[14] Im interkantonalen und internationalen Bereich fallen die Titel oft länger aus als im innerstaatlichen bzw. innerkantonalen Recht. So stammt auch der Erlass der Schweiz mit dem längsten Titel, der auf Deutsch 672 Zeichen umfasst, aus dem Staatsvertragsrecht. Es handelt sich um das

«Abkommen in Form eines Briefwechsels zwischen der Schweizerischen Eidgenossenschaft und der Europäischen Atomgemeinschaft über die Anwendung des Überein-

5 Das «Bundesgesetz betreffend die Überwachung des Post- und Fernmeldeverkehrs» von 2016 (SR 780.1) und das «Bundesgesetz betreffend die Aufsicht über die soziale Krankenversicherung» von 2014 (SR 832.12) sind diesbezüglich atypisch und das Wort «betreffend» wohl ein Versehen. Auch in den Kantonen wird der Begriff «betreffend» nur noch äusserst selten eingesetzt (vgl. aber z. B. das «Gesetz betreffend den Schutz der Bevölkerung» von 2019 des Kantons Zug (BGS 541.1).

6 Aufgrund der Besonderheiten der jeweiligen Syntax ist dies bei Erlassen, die auf Französisch oder Italienisch verfasst werden, nicht möglich.

$7 \quad$ Vgl. z. B. SR 510.107.0 oder NW 312.11

$8 \quad$ Vgl. z. B. SZ 100.100 oder BS 911.610.

$9 \quad$ Vgl. z. B. SR 312.5 oder SR 442.1. 
kommens über die Gründung der Internationalen ITER-Fusionsenergieorganisation für die gemeinsame Durchführung des ITER-Projekts, des Übereinkommens über die Vorrechte und Immunitäten der Internationalen ITER-Fusionsenergieorganisation für die gemeinsame Durchführung des ITER-Projekts und des Abkommens zwischen der Regierung Japans und Euratom zur gemeinsamen Durchführung der Tätigkeiten des breiter angelegten Konzepts im Bereich der Fusionsenergieforschung auf das Hoheitsgebiet der Schweizerischen Eidgenossenschaft» (SR 0.424.111).

[15] Dieser «Monstertitel» kommt in seiner italienischen Fassung auf 674 und in der französischen Version sogar auf beeindruckende 720 Zeichen.

[16] Normalerweise sind die Erlasstitel aber wesentlich kürzer: So beträgt die durchschnittliche Titellänge eines Bundeserlasses (unter Ausschluss des Staatsvertragsrechts) auf Deutsch 82,3, auf Französisch 92,8 und auf Italienisch 90,8 Zeichen. Demgegenüber liegt der Durchschnitt bei Staatsverträgen auf Deutsch bei 150,2, auf Französisch bei 145,8 und auf Italienisch bei 144,1 Zeichen.

[17] Auch in den Kantonen ist ein Unterschied zwischen dem innerkantonalen und dem interkantonalen Recht (d. h. den von den Kantonen untereinander abgeschlossenen Verträgen) sowie dem Staatsvertragsrecht der Kantone (d. h. den von den Kantonen mit dem Ausland abgeschlossenen Verträgen) festzustellen. Die Titel des Vertragsrechts sind regelmässig wesentlich länger (im Durchschnitt über alle Kantone auf Deutsch sind die Titel 117,1 Zeichen, auf Französisch 120,5 und auf Italienisch 106,4 Zeichen lang.)

[18] Im Bereich des innerkantonalen Rechts (ohne interkantonales Recht und Staatsverträge) sind die Unterschiede zwischen den Kantonen verhältnismässig gering und der Durchschnitt variiert zwischen 58 (UR) bis 92 Zeichen (NE):

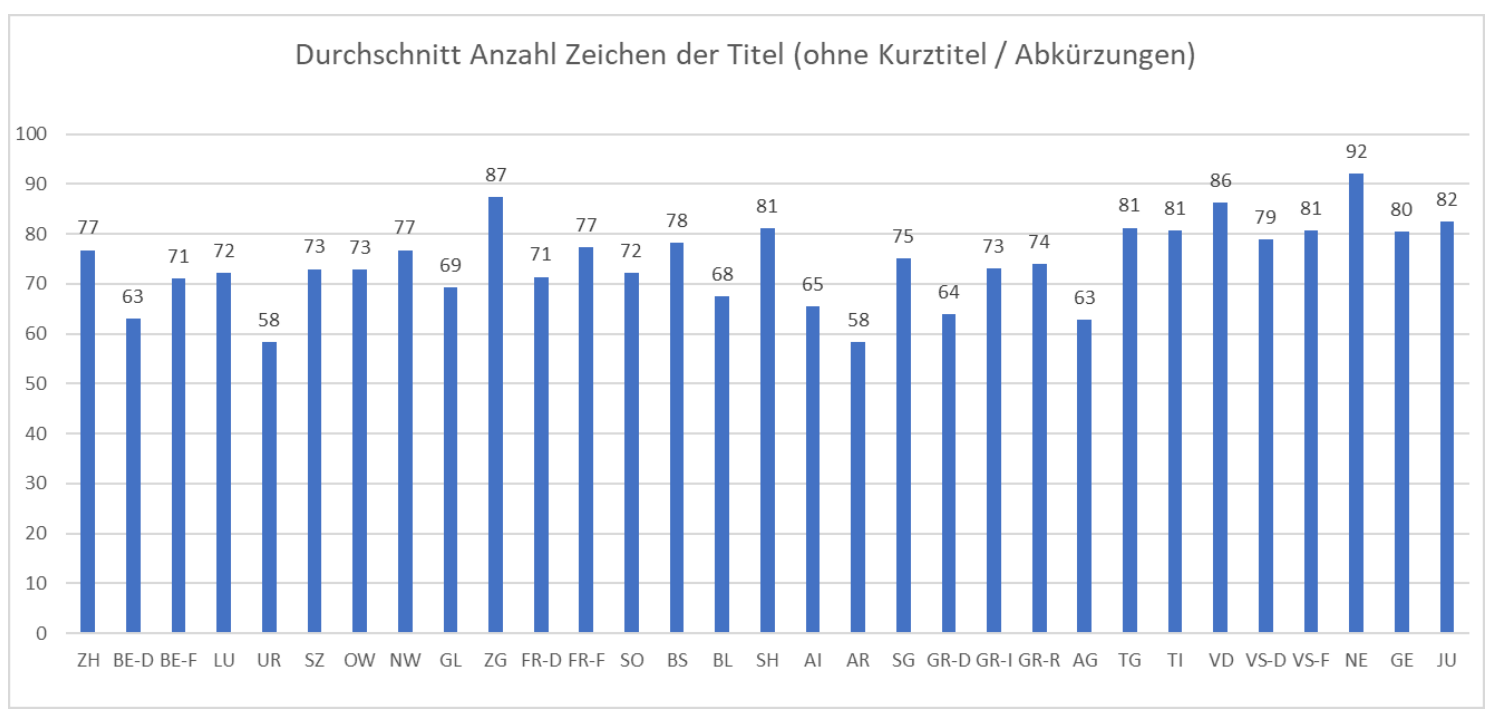

[19] Am anderen Ende der Skala finden sich viele Erlasse mit sehr kurzen Titeln, wie die folgenden Beispiele zeigen: 
[20] Erlasse mit neun oder zehn Zeichen:

\begin{tabular}{|l|l|l|}
\hline Alpgesetz & AI & 916.5 \\
\hline Asylgesetz & CH & 142.31 \\
\hline Baugesetz & AI & 700 \\
\hline Baugesetz & VS & 705.1 \\
\hline Baugesetz & BE & 721 \\
\hline Baugesetz & OW & 710.1 \\
\hline Jagdgesetz & AI & 922 \\
\hline Jagdgesetz & SO & 626.11 \\
\hline Kinogesetz & SG & 554.1 \\
\hline Postgesetz & CH & 783 \\
\hline Waldgesetz & SO & 931.11 \\
\hline Waldgesetz & TG & 921.1 \\
\hline Weggesetz & LU & $758 \mathrm{a}$ \\
\hline Zollgesetz & CH & 631 \\
\hline
\end{tabular}

[21] Erlasse auf Französisch mit zehn bis zwölf Zeichen:

\begin{tabular}{|l|l|l|}
\hline Acte final & $\mathrm{CH}$ & 0.632 .401 .7 \\
\hline Loi de santé & $\mathrm{NE}$ & 800.1 \\
\hline Loi d'impôt & JU & 641.11 \\
\hline Loi fiscale & VS & 642.1 \\
\hline Loi scolaire & VD & 400.01 \\
\hline
\end{tabular}

[22] Erlasse auf Italienisch mit elf bzw. vierzehn Zeichen:

\begin{tabular}{|l|l|l|}
\hline Atto finale & $\mathrm{CH}$ & 0.632 .401 .7 \\
\hline Legge sui cani & TI & 482.3 \\
\hline
\end{tabular}

\section{Abkürzungen und Kurztitel}

\subsection{Allgemeine Betrachtung}

[23] Abkürzungen und Kurztitel sind eng miteinander verwandt: Beide stehen in der Regel in einer Klammer nach dem Erlasstitel und werden mit Komma oder Semikolon getrennt. Sie stellen alternative Zitiermöglichkeiten für Erlassautoren dar. Allerdings ist in der Praxis festzustellen, dass Erlasse fast immer mit dem Langtitel, dem Kurztitel, der Abkürzung, der systematischen Nummer und dem Beschlussdatum zitiert werden, während Abkürzungen erst nach dem erstmaligen Zitat verwendet werden. Ein Vorteil der Abkürzungen besteht jedoch mindestens darin, dass damit in den Erlassportalen von Bund und Kantonen Erlasse sehr rasch gefunden werden können.

[24] Die Verwendung von Kurztiteln und Abkürzungen ist recht häufig. Beim Bund ist ein grosser Unterschied zwischen dem Staatsvertragsrecht und dem Landesrecht festzustellen: Im Staatsvertragsrecht des Bundes sind Abkürzungen eher selten: Nur 67 von insgesamt 2982 Staatsverträgen 
weisen eine Abkürzung oder einen Kurztitel auf. Anders verhält es sich im Landesrecht, wo 1265 von insgesamt 2125 Erlassen über eine alternative Zitiermöglichkeit verfügen.

[25] Spitzenreiter unter den Kantonen ist der Kanton Genf, der jedem Erlass, inkl. Staatsverträgen und interkantonalem Recht, ohne Rücksicht auf dessen Wichtigkeit, eine Abkürzung zugeordnet hat.

[26] Im Bereich des interkantonalen Rechts und des Staatsvertragsrechts sind Abkürzungen ana$\log$ zum Bund ebenfalls weniger häufig: Nur 538 Erlasse der insgesamt von den Kantonen in diesem Bereich publizierten 2152 Erlasse verfügen über eine Abkürzung oder einen Kurztitel.

[27] Die Kantone Jura und Obwalden setzen von allen Kantonen am wenigsten Abkürzungen und Kurztitel ein (JU bei 48 von insgesamt 698 [innerkantonalen] Erlassen, OW bei 51 von insgesamt 437 [innerkantonalen] Erlassen).

[28] Im nachfolgenden Diagramm werden beim Bund nur das Landesrecht, bei den Kantonen nur die innerkantonalen Erlasse unter Ausschluss des interkantonalen Rechts und der Staatsverträge betrachtet.

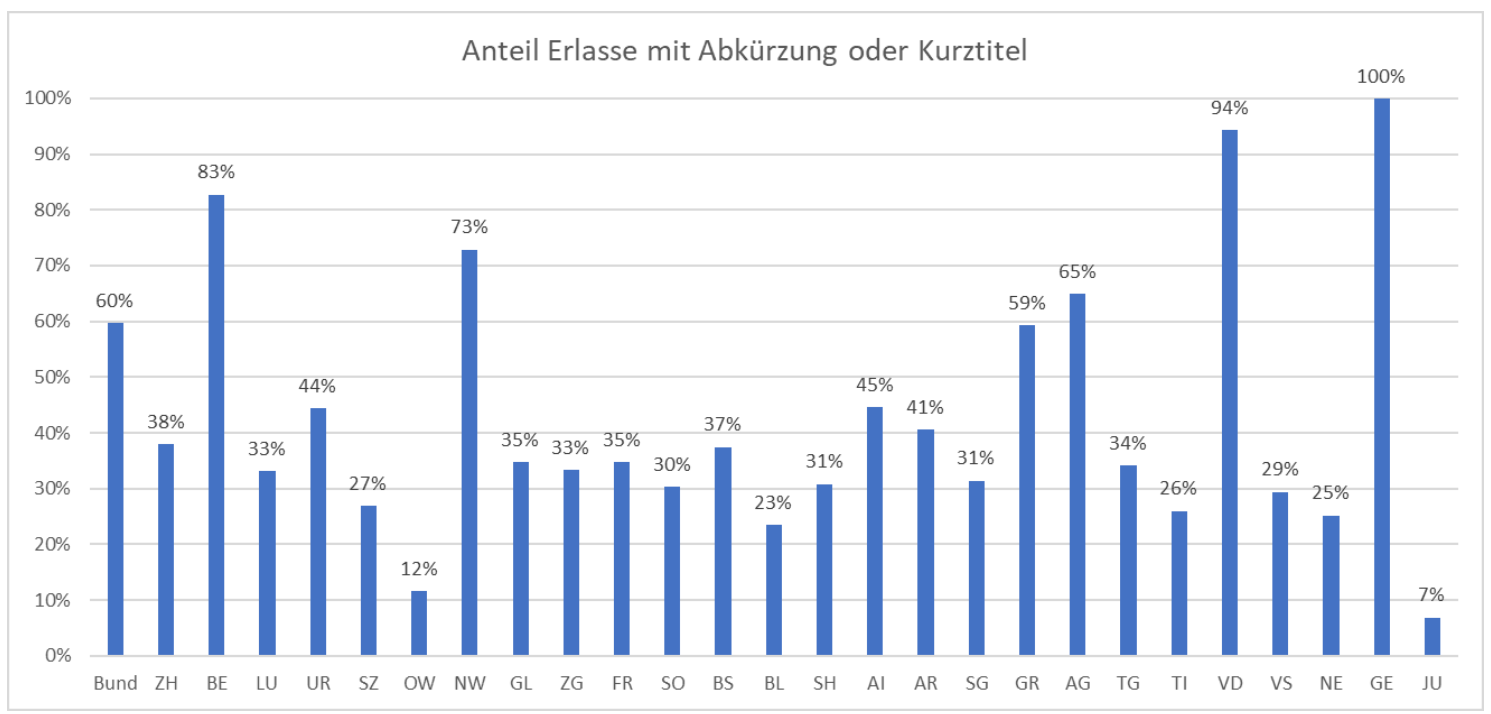

\subsection{Abkürzungen}

[29] Die Abkürzungen stehen in der Regel in einer Klammer hinter dem eigentlichen Erlasstitel. Einzig der Kanton St. Gallen führt seine Leserinnen und Leser in die Irre, indem er zwar Kurztitel in Klammern nach dem Erlasstitel setzt, die Abkürzung jedoch in eine allgemeine Fussnote «verbannt», in welcher auch die Fundstellen vermerkt sind.

[30] Der Bund definiert die Abkürzung in den GTR wie folgt (vgl. Rz. 14, 16 und 17): 
Dem Titel eines Erlasses, von dem anzunehmen ist, dass er besonders häufig zitiert werden wird, kann - evtl. zusätzlich zum Kurztitel - eine Abkürzung beigefügt werden. Dies muss in allen Amtssprachen geschehen. Die Abkürzung wird auf einer neuen Zeile unterhalb des Titels in Klammer angefügt, gegebenenfalls zusammen mit dem Kurztitel; zwischen dem Kurztitel und der Abkürzung steht in diesem Fall ein Komma.

Die Buchstabenkombination sollte aus dem Titel oder dem Kurztitel gebildet werden. Bei der Bildung der Abkürzungen ist darauf zu achten, dass ein Grossbuchstabe zu verwenden ist, wenn das damit abgekürzte Wort einen eigenständigen Begriff bildet (z. B. OR, BV). Dem Grossbuchstaben können auch Kleinbuchstaben folgen (z. B. StGB). Zwischen den Buchstaben stehen keine Punkte.

Die Abkürzung besteht aus höchstens fünf Buchstaben.

[31] Eine ähnliche Praxis besteht auch im Kanton Zürich ${ }^{10}$ :

Ist zu erwarten, dass ein Erlass in der Praxis häufig zitiert wird, kann in Klammern eine Abkürzung beigefügt werden. Die Abkürzung soll aus höchstens fünf Buchstaben bestehen, wobei die Buchstabenkombination dem ausgeschriebenen Haupttitel entnommen wird. Dabei wird ein Grossbuchstabe verwendet, wenn das damit abgekürzte Wort einen eigenständigen Begriff bildet, z. B. StPO für Strafprozessordnung.

Bereits bestehende Abkürzungen des Zürcher Rechts und die bekannten, häufig verwendeten Abkürzungen des Bundesrechts dürfen nicht nochmals verwendet werden. Über die Abkürzungen von Erlasstiteln des Bundesrechts informiert das von der Bundeskanzlei periodisch nachgeführte Abkürzungsverzeichnis «Amtliche Abkürzungen des Bundes» (Bezugsstelle [...]). Die bereits verwendeten Abkürzungen des Zürcher Rechts finden sich zu Beginn jedes Bandes der LS.

[32] Nicht jede Klammerbemerkung in einem Titel stellt auch die Abkürzung des jeweiligen Erlasses dar. Dies kann in der Praxis leicht zu Verwechslungen führen. So ist beispielsweise der Titel «Gesetz über die Pensionskasse das Kantons Wallis (PKWAL)» ${ }^{11}$ verwirrend, weil «PKWAL» unter Umständen für die Abkürzung des Erlasses gehalten werden könnte; dabei handelt es sich richtigerweise um die Abkürzung der im Titel genannten Organisation. Verwirrend ist auch der Titel «Interkantonale Fachhochschulvereinbarung (FHV) ab 2005» weil die (potenzielle) Abkürzung (FHV) nicht am Ende des Titels steht. Konfus können ausserdem kantonale Einführungsgesetze zu Bundesgesetzen sein, welche die Abkürzung des Bundeserlasses wiederholen, wie z. B. das «Einführungsgesetz zum Bundesgesetz über den Binnenmarkt (BGBM)» des Kantons Schaffhausen (SH 172.5). Auch hier könnte eine unbedarfte Leserin oder ein unbedarfter Leser die Klammerbemerkung als Abkürzung des Erlasses missverstehen.

10 Vgl. Richtlinien der Rechtsetzung vom 21. Dez. 2005, Rz. $31 \mathrm{f}$.

11 VS 172.51 
[33] Die Abkürzung setzt voraus, dass sie eindeutig ist, d. h. zur gleichen Zeit sollte es im gleichen Kanton bzw. im Bund nur einen Erlass geben, der über dieselbe Abkürzung verfügt. Die Eindeutigkeit ist in den Kantonen und im Bund in aller Regel gegeben, wobei es aber auch Beispiele mit mehrdeutigen Abkürzungen gibt. So steht beispielsweise im Kanton Waadt die Abkürzung «RPCL» sowohl für das «Règlement sur l'utilisation des pompes à chaleur» (VD 730.05.1) als auch für das «Règlement sur les prêts et les cautionnements pour les logements» (VD 840.11.5).

[34] Das auf Bundesebene bestehende Gebot, dass Abkürzungen in allen Amtssprachen vorhanden sein müssen, klappt in den (mehrsprachigen) Kantonen gut. Im Bund existieren nur wenige

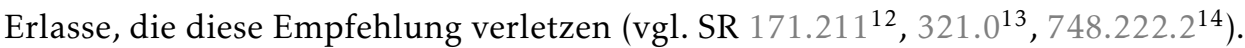

[35] Bei Abkürzungen sollte schliesslich auch darauf geachtet werden, dass sie das Zitieren von Erlassen vereinfachen (und nicht erschweren) sollten. Daher sollten sie - um ihren Zweck zu erfüllen - leicht ausgesprochen und im Gedächtnis behalten werden können. So gesehen muten Abkürzungen wie RISBPC $^{15}$, $«$ KAMLR ${ }^{16}$, «RCoFLAA» ${ }^{17}$, «RaORRChim» ${ }^{18}$, «VVzEGzAHVG/IVG» ${ }^{19}$ oder «RRFMOI» ${ }^{20}$ eher kryptisch an.

\subsection{Kurztitel}

[36] Kurztitel erfüllen im Grunde genommen denselben Zweck wie Abkürzungen. Auch sie dienen dazu, Erlasse leichter bzw. mit weniger Text zitieren zu können. Sie werden ebenfalls in der Klammer nach dem Titel gesetzt. Die Kurztitel stehen in einer gewissen Konkurrenz zum Langtitel, denn es erscheint nicht immer nachvollziehbar, weshalb noch ein Langtitel nötig sein soll, wenn bereits ein prägnanter und verständlicher Kurztitel möglich ist. Beispielsweise ist der Langtitel «Gesetz über die Mittelschulen im Kanton Graubünden» ${ }^{21}$ überflüssig, wenn gleichzeitig der Kurztitel «Mittelschulgesetz» dasselbe aussagt. Die Praxis ist in den Kantonen jedenfalls uneinheitlich: «Schulgesetz» wird in den Kantonen ZG, BS, SH und $\mathrm{AG}^{22}$ als Langtitel verwendet und in den Kantonen UR, NW, FR, AR und GR ${ }^{23}$ (lediglich) als Kurztitel.

12 Verordnung der Bundesversammlung zum Parlamentsressourcengesetz (VPRG) | Ordonnance de l'Assemblée fédérale relative à la loi sur les moyens alloués aux parlementaires (OMAP) | Ordinanza dell'Assemblea federale concernente la legge sulle indennità parlamentari.

13 Militärstrafgesetz (MStG) | Code pénal militaire (CPM) | Codice penale militare.

14 Verordnung des UVEK über die JAR-FCL-Lizenzen zum Führen von Flugzeugen und Hubschraubern (VJAR-FCL) | Ordonnance du DETEC sur les titres de vol JAR-FCL pour pilotes d'avion et d'hélicoptère (OJAR-FCL) | Ordinanza del DATEC concernente le licenze JAR-FCL per piloti d'aeroplano e d'elicottero.

15 Règlement provisoire fixant le taux d'imposition à la source pour certains bénéficiaires de participations de collaborateur domiciliés à l'étranger (GE D 3 20.04).

16 Reglement der Konsultativkommission für die Ausbildung in Milchwirtschaft und Lebensmitteltechnologie (FR 911.11.71).

17 Règlement de la Commission consultative pour la formation laitière et agro-alimentaire (FR 911.11.71).

18 Règlement d'application de l'ordonnance fédérale sur la réduction des risques liés aux produits chimiques (GE K 1 70.12).

19 Vollziehungsverordnung zum Einführungsgesetz zu den Bundesgesetzen über die Alters- und Hinterlassenenversicherung und die Invalidenversicherung (GR 544.010).

20 Règlement sur le registre foncier et la mensuration officielle informatisés (GE E 1 50.08).

21 Gesetz über die Mittelschulen im Kanton Graubünden (Mittelschulgesetz, MSG; GR 425.000).

22 ZG 412.11, BS 410.1, SH 410.1, AG 401.1.

23 Vgl. UR 10.1111: Gesetz über Schule und Bildung; NW 312.1: Gesetz über die Volksschule; FR 411.0.1: Gesetz über die obligatorische Schule; AR 411.0: Gesetz über Schule und Bildung; GR 421.000: Gesetz für die Volksschulen des Kantons Graubünden. 
[37] Mit anderen Worten besteht keine Pflicht, einen «Langtitel» zu vergeben, wenn bereits ein «Kurztitel» genügt. In diesem Sinn sind Erlasse wie «Spitalverordnung» ${ }^{24}$, «Zeitgesetz» ${ }^{25}$ oder «Ombudsgesetz» ${ }^{26}$ gute Beispiele für prägnante Gesetzgebung.

[38] In den GTR des Bundes findet sich folgende Definition der Kurztitel (vgl. Rz. 10 und 11):

Ein Kurztitel soll das Zitieren des Erlasses erleichtern. Nicht jeder Erlass braucht einen Kurztitel; man wählt neben dem Titel einen Kurztitel in der Regel dann, wenn es sich um einen häufig zitierten Erlass handelt, sein Titel lang ist und der Kurztitel eine erhebliche Verkürzung gegenüber dem Titel bedeutet. Der Kurztitel wird auf einer neuen Zeile unter dem Titel in Klammern beigefügt. Hat ein Erlass einen Kurztitel, so wird zum Zitieren nur dieser verwendet.

[39] Auch bei Kurztiteln ist darauf zu achten, dass sich die Fassungen in den verschiedenen Amtssprachen entsprechen, wobei Kurztitel in Form eines zusammengesetzten Substantivs (z. B. "Gewässerschutzgesetz») nur auf Deutsch möglich sind. Anders als bei Abkürzungen (vgl. GTR des Bundes, Rz. 14) ist es nach den Richtlinien zulässig, dass ein Erlass nicht in allen Amtssprachen einen Kurztitel trägt.

[40] Aus den Kantonen können beispielsweise die «Richtlinien des Kantons Thurgau für die Gesetzgebung (Ausgabe 2004)» erwähnt werden, die im Kapitel 3.2 Folgendes zu den Kurztiteln festhalten:

Erlasse können Kurztitel tragen. Ein solcher ist vor allem dann sinnvoll, wenn der Erlasstitel lang ist. Der Kurztitel wird in Klammern beigefügt.

Kurztitel sind in der Praxis äquivalent zu Titeln. Sie stellen i.d.R. «sprechendere Abkürzungen» oder schlicht ausformulierte Abkürzungen dar.

[41] Damit Kurztitel ihre Wirkung entfalten können, d. h. zu einer tatsächlichen Erleichterung bei der Zitierung führen, sollten sie möglichst kurz sein bzw. sollten die Langtitel über eine gewisse Länge verfügen, damit ein Kurztitel sinnvoll erscheint.

[42] Der Kurztitel «Adoptionsverordnung» für den Erlass «Verordnung über die Adoption» ${ }^{27}$ erfüllt diesen Anspruch m. E. ebenso wenig wie "Archivverordnung» für die «Verordnung zum Archivgesetz» ${ }^{28}$ oder «Abfallgesetz» für das «Gesetz über die Abfälle» ${ }^{29}$. Bei diesen Titeln stellt sich eher die Frage des Mehrwerts des Langtitels gegenüber dem Kurztitel als umgekehrt.

[43] Prägnante Wortkombinationen, wie sie auf Deutsch möglich sind, lassen sich in der französischen und italienischen Sprache nicht (immer) bilden. Insofern haben die Amtssprachen Französisch und Italienisch hinsichtlich Kurztitel einen Nachteil gegenüber der deutschen Sprache. Deshalb sind dort Kurztitel teilweise nur beschränkt sinnvoll. Ein gutes Beispiel dafür ist SR 950.1

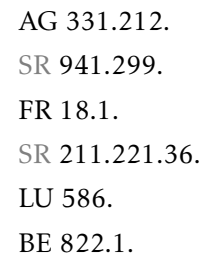


in der italienischen Version «Legge federale sui servizi finanziari», welcher als Kurztitel «Legge sui servizi finanziari» trägt (der Unterschied beschränkt sich auf das Weglassen des Worts «federale»). Auch in solchen Fällen sollte der Kurztitel weggelassen werden, da er keine wesentliche Verkürzung des Titels zur Folge hat.

[44] In den Kantonen, die nur Französisch oder Italienisch als Amtssprache haben, sind (wohl auch aus diesem Grund) Kurztitel nicht besonders häufig; nur gerade 27 Erlasse tragen einen solchen Titel (so z. B. «Loi sur l'exploitation des matières premières minérales [Loi sur les mines]» JU 931.1 oder «Legge cantonale sui provvedimenti per combattere le epizoozie [legge sulle epizoozie]» TI 914.400).

[45] Analog zu Abkürzungen und Titeln sollten auch Kurztitel eindeutig sein, damit sie sich für das Zitieren eignen. Tragen mehrere Erlasse denselben Kurztitel, ist das Zitieren allein mit dem Kurztitel nicht mehr möglich. Der Bund führt derzeit acht Staatsverträge unter dem Kurztitel «Rückübernahmeabkommen»; aus den Kantonen können namentlich der Kanton Luzern erwähnt werden, der gleich viermal einen Erlass mit dem Kurztitel «Fakultätsreglement» und der Kanton Zürich, der sieben Erlasse mit dem Kurztitel «Promotionsverordnung» führt.

[46] Auch müssen Kurztitel nicht zwingend aus Wörtern oder Wortbestandteilen des Langtitels bestehen (vgl. z. B. «Bundesgesetz über die Bekämpfung übertragbarer Krankheiten des Menschen (Epidemiengesetz, EpG»; SR 818.101).

[47] Schliesslich sei noch erwähnt, dass die Unterscheidung zwischen einer Klammerbemerkung am Ende des Titels und einem Kurztitel in der Praxis missverständlich sein kann. Beispielsweise ist unklar, ob die Klammerbemerkung «Etzelwerkkonzession» bei der «Vereinbarung zwischen dem Kanton Schwyz, vertreten durch den Regierungsrat, und den Schweizerischen Bundesbahnen, vertreten durch ihre Generaldirektion in Bern, zum Vertrag über die Ausnützung der Wasserkräfte der SihI beim Etzel (Etzelwerkkonzession)» (SRSZ 452.110.2) einen Kurztitel darstellt oder zum eigentlichen Titel gehört. Als weitere Beispiele können die «Verordnung über die Gemeinen Alpen (Alpbüchlein)» (AI 916.520) oder die «Ordnung für den Preis der Universität Basel (Amerbach-Preis)» (BS 494.400) genannt werden. Auch in diesen Fällen ist nicht klar, ob die in Klammern gesetzten Begriffe Kurztitel darstellen sollen.

[48] Teilweise sind Kurztitel auch schlicht zu lang, wie die folgenden Beispiele zeigen:

- «Verordnung über die Branchen- und Produzentenorganisationen» (SR 919.117.72),

- «Verordnung über das Inverkehrbringen von Produkten nach ausländischen Vorschriften» (SR 946.513.8),

- «Prüfungsverordnung für die Abschlussprüfungen der Fachmittelschule FMS» (SO 414.134).

[49] Kann kein (sinnvoller) prägnanter und unmissverständlicher Kurztitel gefunden werden, sollte darauf verzichtet werden. Ein solches Beispiel stellt das «Bundesgesetz über die Vermittlung Schweizer Bildung im Ausland» (SR 418.0) dar, dessen Kurztitel «Schweizerschulengesetz» lautet. Allein aus dem Kurztitel lässt sich kaum herauslesen, dass das Gesetz die Unterstützung der Schweizer Schulen im Ausland und die Vermittlung schweizerischer Ausbildung im Ausland zum Gegenstand hat (vgl. Art. 1). Gleich verhält es sich mit dem «Dekret über die Überführung der Personalvorsorge für Lehrpersonen an der Volksschule in die Aargauische Pensionskasse (Überführungs-Dekret)»; AG 413.310. Auch hier ist der Kurztitel «Überführungs-Dekret» wenig aussagekräftig. 


\section{Titel von Geschäften/Publikationen in der chronologischen Gesetzes- sammlung}

[50] Von den bisher diskutierten Erlasstiteln der systematischen Sammlungen zu unterscheiden sind die Titel der Geschäfte, d.h. der Beschlüsse, die in der chronologischen Sammlung publiziert werden und zum Zweck haben, dass ein oder mehrere Erlasse der systematischen Sammlung neu verabschiedet, geändert oder aufgehoben werden. Mit anderen Worten beschreiben die Geschäfte stets eine Mutation in der Gesetzessammlung. Aus diesem Grund sollten diese Mutationsbeschreibungen auch anders benannt werden als die entsprechenden Erlasse.

[51] Der Bund behandelt diese Titel nicht anders als die übrigen Erlasstitel (vgl. GTR, Rz. 282):

Als Titel des Änderungserlasses wird der unveränderte Titel des zu ändernden Erlasses einschliesslich des allfälligen Kurztitels und der allfälligen Abkürzung aufgeführt.

[52] Somit kann einem Titelverzeichnis der chronologischen Sammlung nicht entnommen werden, ob es sich um eine Änderung, eine Aufhebung, einen neuen Erlass oder eine Kombination davon handelt. Erst beim Öffnen des entsprechenden Dokuments wird die Art der Änderung ersichtlich.

[53] Viele Kantone (vgl. z. B. ZH, BE, SO, BL) handhaben dies anders und erstellen eigenständige Titel für Geschäfte (Änderungserlasse) bzw. merken dort an, um was für eine Mutationsart es sich handelt.

[54] Hilfreich sind ausserdem Inhaltsverzeichnisse in den Geschäften, die Auskunft darüber geben, welche Erlasse von einem Geschäft betroffen sind. Solche Inhaltsverzeichnisse kennen beispielsweise die Kantone BE, ZG, FR oder VS, wie das folgende Beispiel zeigt:

\section{Gesetz}

über den Justizrat

(GJR)

vom 13.09.2019

Von diesem Geschäft tangierte Erlasse (SGS Nummern)

Neu: $\quad 173.7$

Geändert: $\quad 160.5$ | 171.1 | 173.1 | 312.0 | 611.1

Aufgehoben: - 


\section{Die Änderung von Titeln und Abkürzungen}

\subsection{Allgemeine Betrachtung}

[55] Wie bereits erwähnt, dienen Titel und Abkürzungen hauptsächlich der Auffindbarkeit der Rechtsnormen und deren Zitierung. Werden Titel oder Abkürzungen geändert, werden die Auffindbarkeit der Erlasse und die richtige bzw. eindeutige Zitierung erschwert. Damit haben Änderungen der Titel oder der Abkürzungen indirekt Auswirkungen auf die Auffindbarkeit der zu einer bestimmten Rechtsnorm massgebenden Rechtsprechung oder Lehre, weil auch Gerichte, verfügende Behörden und Autoren von Fachliteratur die Erlasse mit Titel, Kurztitel und Abkürzung zitieren.

[56] Die Änderung von Titeln und Abkürzungen ist aber ein durchaus üblicher Vorgang. Seit 2018 wurden in der Schweiz bei 376 Erlassen (Erlassversionen) Titel oder Abkürzungen angepasst (von insgesamt 8506 Teilrevisionen). Bei Totalrevisionen sind die Titel- und Abkürzungsänderungen sehr viel häufiger: Dort änderte seit 2018 von 852 Erlassen, die von einer Totalrevision betroffen waren, bei 646 Erlassen auch der Titel oder deren Abkürzung.

[57] Es stellt sich somit die Frage, wer Titel und Abkürzungen ändern darf und in welchen Fällen dies gemacht bzw. lieber darauf verzichtet werden sollte.

\section{2. Änderungen von Titeln}

[58] Titel werden vom Bund und von den Kantonen grundsätzlich gleichbehandelt wie der normale Erlasstext, d. h. auch die Titel stehen zur Disposition des politischen Diskurses und werden im Rechtsetzungsprozess geändert.

[59] Weil Titeländerungen, wie bereits erwähnt, die Auffindbarkeit der Erlasse erschweren, und Erlasse üblicherweise mit ihrem Titel zitiert werden, sollte dies nur zurückhaltend und aus triftigen, sachlichen Gründen erfolgen. Ein solcher Grund kann beispielsweise in einer veralteten Terminologie bestehen, die aus aktueller Sicht nicht mehr vertretbar ist. ${ }^{30}$ Leichtfertig sollten Titeländerungen jedoch nicht erfolgen.

[60] Ein Beispiel dafür ist das «Bundesgesetz über die Ausländerinnen und Ausländer» (SR 142.20), das seit dem 1. Januar 2019 neu «Bundesgesetz über die Ausländerinnen und Ausländer und über die Integration» heisst. Die Botschaft führt zur Titeländerung aus, dass diese erfolge, «um die gesellschaftliche Bedeutung der Integration zu unterstreichen». ${ }^{31}$ Es mag zwar verständlich sein, dass der Gesetzgeber den Wunsch hat, eine Titeländerung mit einer politischen Botschaft zu verknüpfen, doch bildet dies nach Auffassung des Autors noch keinen hinreichend gewichtigen Grund, um die eindeutige Identifizierung und das Auffinden eines Erlasses zu erschweren. Dies insbesondere auch deshalb, weil die Förderung der Integration von Ausländerinnen und Ausländern schon vor der erfolgten Titeländerung Regelungsgegenstand des Gesetzes (vgl. Art. 1 in der bis Ende 2018 geltenden Fassung des AIG) war.

30 Beispielsweise war im «Vertrag zwischen den Kantonen Appenzell I.Rh. und Appenzell A.Rh. über die Aufnahme psychisch Kranker aus dem Kanton Appenzell I.Rh. in die Kantonale Psychiatrische Klinik in Herisau» (AR 812.26) bis 2008 in Art. 1 von «Altersblödsinn» die Rede. Heute könnte man sich beispielsweise auch fragen, ob die Terminologie «invalid» noch angebracht ist. 
[61] Anders wäre es, wenn Erlasse nur noch mit der systematischen Nummer zitiert würden. In diesem Fall würden die Titel nur noch eine untergeordnete Rolle spielen und könnten so den aktuellen politischen Willen wiedergeben. Zu bemerken ist aber, dass auch systematische Nummern ändern können. So führte beispielsweise der Kanton Tessin im Jahr 2018 eine komplett neue Nummerierung der Erlasse ein, und auch sonst wurde seit 2018 bei gesamtschweizerisch 12 Erlassen die Nummer gewechselt. ${ }^{32}$

[62] Bei Totalrevisionen wird sehr häufig der Titel und oder die Abkürzung geändert. Auch in diesen Fällen sollte nach Möglichkeit darauf geachtet werden, dass der Erlasstitel nicht geändert wird, da ansonsten das «Wiederfinden» des Nachfolgeerlasses erschwert wird. Dieses Kriterium wirkt bei Totalrevisionen weniger stark als bei Teilrevisionen, da bei Ersteren der eigentliche Inhalt und die Artikelstruktur (komplett) neu festgelegt werden.

[63] Titeländerungen können auch durch Wechsel der Erlassform entstehen: So wurde der «Bundesbeschluss über die Gewährung von Zollpräferenzen zugunsten der Entwicklungsländer (Zollpräferenzenbeschluss)» (SR 632.91) per 1. März zum «Bundesgesetz über die Gewährung von Zollpräferenzen zugunsten der Entwicklungsländer (Zollpräferenzengesetz)» umbenannt. Gleiches passierte beim «Bundesbeschluss über die Zusammenarbeit mit den Internationalen Gerichten zur Verfolgung von schwerwiegenden Verletzungen des humanitären Völkerrechts» (SR 351.20), der per 1. Januar 2009 zum «Bundesgesetz über die Zusammenarbeit mit den Internationalen Gerichten zur Verfolgung schwerwiegender Verletzungen des humanitären Völkerrechts» wurde. Ähnliche Beispiele finden sich auch in den Kantonen, so z. B. im Kanton Schwyz, wo namentlich die «Verordnung über die Besoldung der Behörden und das Dienstverhältnis des Staatspersonals» (SZ 140.510), per 1. Januar 2014 zum «Gesetz über die Besoldung der Behörden und das Dienstverhältnis des Staatspersonals» wurde. Diese Praxis wird mit der Angleichung der Erlassform an die jeweils aktuelle Verfassung begründet. ${ }^{33}$ Nach Meinung des Autors kann jedoch die Erlassform nicht durch blosse Titeländerung, sondern nur durch Aufhebung und Verabschiedung eines neuen Erlasses (Totalrevision) modifiziert werden. Entscheidend für die Bezeichnung der Erlassform ist stets das bei der Verabschiedung angewendete Verfahren. Mit der Titeländerung kann somit nicht die Form bzw. das Wesen des Erlasses, sondern höchstens dessen Bezeichnung geändert werden. Formell bleiben die Erlasse aber «Bundesbeschlüsse» bzw. «Verordnungen».

[64] Schliesslich ist auch auf die fehlgeleitete Praxis hinzuweisen, Jahresangaben in Titeln festzuhalten, wie es in den folgenden Beispielen der Fall ist:

- «Verordnung ILFD über die Planung der Jagdsaison 2020» (FR 921.111)

- «Verordnung zur Prämienverbilligung für das Jahr 2020» (NW 742.111)

- «Arrêté fixant les paramètres applicables en matière de péréquation financière pour l'année 2020» (JU 651.111)

[65] Solche Erlasse werden jährlich totalrevidiert und für das jeweils neue Jahr neu verabschiedet. Bei genauerer Betrachtung fällt jedoch auf, dass oft nur einzelne wenige Parameter geändert wer-

32 Zuletzt gerade im Okt. 2020 beim Bund beobachtet, indem SR 442.17 nach SR 442.15 verschoben wurde.

33 Vgl. BBl 2006 2968; BBl 2007 7538; SZ GS 23-80 (Kantonsratsbeschluss betreffend die Anpassung des Gesetzes über die Organisation der Gemeinden und Bezirke und weiterer Erlasse an die Verfassung des Kantons Schwyz vom 24. Nov. 2010). 
den, während die Erlassstruktur eigentlich gleichbleibt. Deshalb wäre es an sich einfacher, auf die Jahresangabe im Titel zu verzichten und solche Erlasse jeweils teilzurevidieren. Ausnahmsweise können Jahresangaben bei befristeten Erlassen (z. B. bei Notrecht) Sinn machen.

\section{3. Änderung von Abkürzungen}

[66] Abkürzungen werden von Bund und Kantonen ähnlich betrachtet wie Titel und werden von den politischen Gremien entsprechend eingeführt oder geändert. Dies ist aus der Sicht des Autors falsch: Der Sinn und Zweck von Abkürzungen besteht lediglich darin, die Zugänglichkeit von Erlassen zu erleichtern. Noch weniger als im Titel kann in der Abkürzung ein normativer Inhalt oder ein politisches Anliegen verpackt werden; vielmehr handelt es sich um einen Vorschlag für eine mögliche Alternativzitierung.

[67] Aus diesem Grund ähneln Abkürzungen eher den systematischen Nummern als den Titeln und sollten deshalb, wie die systematischen Nummern auch, von der für die Erlasspublikation zuständigen Stelle nach einheitlichen Kriterien vergeben werden. Dies wird vereinzelt auch so gehandhabt, wenn z. B. die Staatskanzlei bei Erlassen mittels formloser Berichtigung Abkürzungen ergänzt, wie sie das beispielsweise bei nachfolgenden Erlassen vorgenommen hat: TG 131.1 und 551.1, VS 935.52 oder FR 638.1.

[68] Da Abkürzungen ohnehin ein künstliches Produkt darstellen, sollten sie mit noch grösserer Zurückhaltung geändert werden als Titel. Im oben genannten Beispiel des Bundesgesetzes über die Ausländerinnen und Ausländer und über die Integration wäre es, aus der Sicht des Autors, mit Blick auf die Auffindbarkeit sinnvoller gewesen, die bisherige Abkürzung «AuG» beizubehalten, anstatt mit der neuen Abkürzung «AIG» zu versuchen, möglichst nahe beim Titel zu bleiben.

[69] Allenfalls könnte man auch zum Schluss kommen, dass - neben den Abkürzungen und den systematischen Nummern - auch die Erlasstitel keine normativen Inhalte vermitteln, sondern reine Beschreibungen des Inhalts sind. Damit würden auch die Erlasstitel nicht zur Disposition der Politik stehen und - ähnlich wie andere Formdefinitionen wie Strukturierungsvorgaben, Artikel- bzw. Paragrafennummerierung, systematische Nummer, aber auch das Papierformat von der für die Erlasspublikation zuständigen Stelle vergeben werden sollten.

[70] Auch hier besteht das Argument in der Trennung zwischen Form und Inhalt: Die Politik kann den normativen Inhalt des Rechts frei definieren. Die Form, die für den Inhalt nicht relevant ist, kann anderweitig vorgegeben bzw. verwaltet und auf die Bedürfnisse der Rechtssuchenden sowie der Rechtsanwenderinnen und Rechtsanwender optimiert werden.

\section{Zitierung von Erlassen}

[71] Wie erwähnt, ändern sich Titel von Erlassen schneller, als man denken könnte. Auch die Rechtsgrundlagen ändern sich rascher, als man es sich grösstenteils bewusst ist. So wurde beispielsweise die «Verordnung über die Einfuhr von landwirtschaftlichen Erzeugnissen» (SR 916.01) seit 2006 schon über 250 Mal revidiert, und die «Verordnung des BLV über Massnahmen zur Verhinderung der Einschleppung der Aviären Influenza aus bestimmten Mitgliedstaaten der Europäischen Union» (SR 916.443.102.1) wurde allein im Zeitraum zwischen dem 1. Januar und dem 31. Oktober 2020 bereits 20 Mal geändert. 
[72] Bei der Zitierung von Erlassen geht es immer darum, die Leserin oder den Leser bzw. die Rechtsanwenderin oder den Rechtsanwender in die Lage zu versetzen, einen Text oder eine Passage einfach und effektiv nachschlagen zu können. Insbesondere für rechtsanwendende Behörden ist die Zitierung bei der Redaktion von Entscheiden zentral.

[73] Es fragt sich deshalb, auf welche Weise erreicht werden kann, dass Lesende möglichst einfach einen zitierten Erlass nachschlagen können.

[74] Wie bereits erwähnt, ist die systematische Nummer der Erlasse die (derzeit) stabilste, beständigste Angabe. Deshalb ist diese bedeutender als der volatile Titel oder die Abkürzung. Wichtig ist in der heutigen Zeit, in der das Recht rasch und häufig ändert, auch die Angabe der durch den Zitierenden verwendeten Fassung. Dafür eignet sich das «Stand-Datum», welches Auskunft darüber gibt, wann die letzte Änderung im jeweiligen Dokument in Kraft trat. Durch die Kombination von systematischer Nummer und Stand-Datum kann ein Erlass - mit Ausnahme von rückwirkenden Inkrafttreten - relativ präzise identifiziert werden. Demgegenüber eignen sich Titel oder Abkürzungen, die jederzeit ändern können, für eine Zitierung nur bedingt.

[75] Auch die heute weit verbreitete Praxis, Erlasse (auch) mit dem Beschlussdatum zu zitieren, kann unpräzise sein und hilft der Leserin oder dem Leser kaum weiter, weil dadurch nur die jeweilige Erstverabschiedung des jeweiligen Erlasses bzw. eine spätere Totalrevision referenziert wird, nicht aber die konkrete Fassung. So erfährt beispielsweise ein Hinweis auf eine Bestimmung des ZGB keinen Mehrwert bzw. keine weitere Präzisierung, wenn noch das Beschlussdatum (10. Dez. 1907) angegeben wird.

\section{Empfehlungen}

[76] Aus der Sicht eines Praktikers im Umgang mit der Entwicklung des Rechts können folgende Empfehlungen ans Herz gelegt werden:

1. Es sollten immer möglichst kurze (Lang-)Titel verwendet werden. Falls ein Kurztitel den Regelungsgegenstand genügend beschreibt, kann auch bloss der Kurztitel als Langtitel verwendet werden.

2. Fussnoten sollten in Titeln, Kurztiteln und Abkürzungen vermieden werden.

3. Klammerbemerkungen in Titeln sollten vermieden und Klammern nur für Kurztitel und Abkürzungen verwendet werden.

4. In Titeln sollten keine Jahresangaben verwendet werden.

5. Kurztitel sollten auch kurz sein, ansonsten sollte darauf verzichtet werden.

6. Nicht jeder Erlass benötigt eine Abkürzung.

7. Abkürzungen sollten einfach im Gedächtnis behalten und auch ausgesprochen werden können, ansonsten verfehlen sie das Ziel.

8. Bei Geschäften (Änderungserlassen) sollte im Titel auch die Mutationsart angegeben werden.

9. Auf Titeländerungen sollte immer verzichtet werden, ausser es bestehen triftige Gründe.

10. Abkürzungen sollten nicht von den politischen Instanzen, sondern von der mit der Publikation der Erlasse zuständigen Stelle einheitlich vergeben und anschliessend nicht mehr geändert werden. 
11. Erlasse sollten immer mit der systematischen Nummer und dem Standdatum zitiert werden. Der blosse Titel oder die Abkürzung reichen aufgrund der Möglichkeit der Titeländerung nicht aus.

Marius Roth, Dr. iur., Direktor des Zentrums für Rechtsinformation - ZRI, Zürich, und Projektleiter LexFind www.lexfind.ch, marius.roth@zri.ch. 